\title{
DISAPPEARANCE OF PATHOLOGICAL Q WAVES AFTER CARDIAC INFARCTION
}

\author{
BY \\ M. P. PAPPAS* \\ From the Department of Cardiology, the Middlesex Hospital \\ Received June 6, 1957
}

Experimental ligation of a branch of a coronary artery has shown that the affected part of the myocardium passes through a number of stages, namely ischæmia, injury, and necrosis, the latter producing Q or QS waves in electrocardiographic leads taken over the necrosed area. If this dead area is transmural, it does not react to the stimulus of contraction and leads from points over it simply reflect the intracavity potential. The earlier stages, ischæmia and injury, are responsible for changes in the part of the electrocardiogram that is due to repolarization, i.e. the R-T segment and T

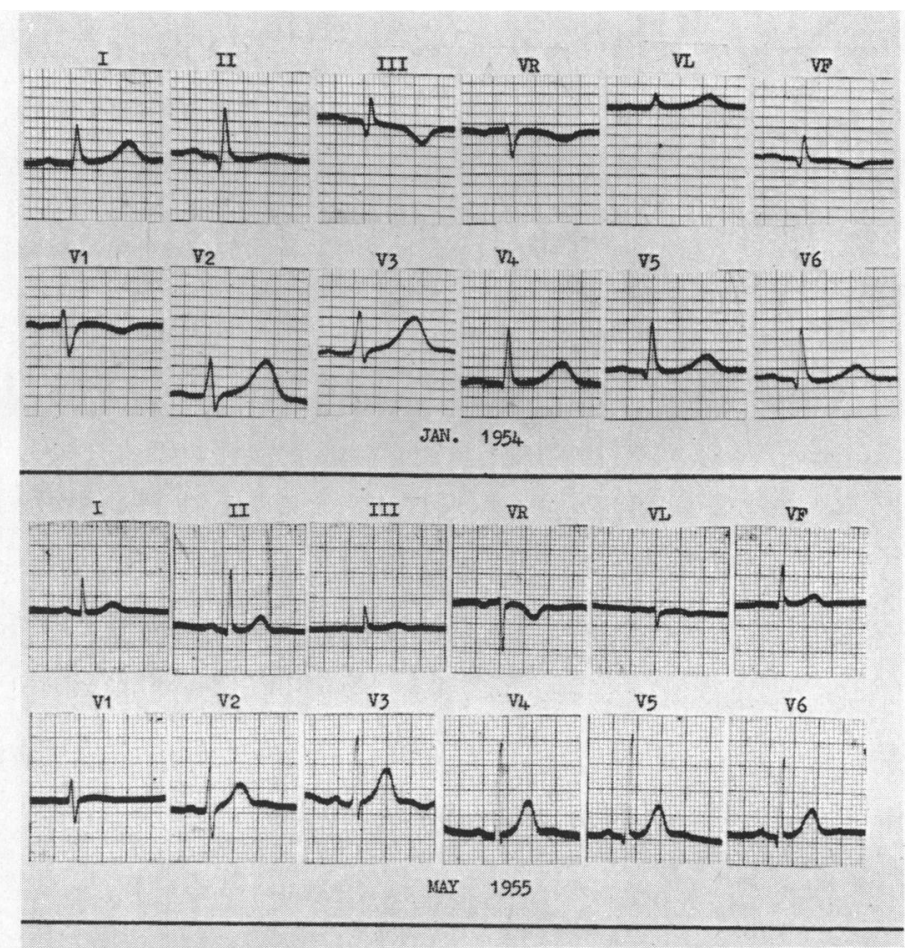

FIG. 1.-Cardiogram showing posterior cardiac infarction(January, 1954). In the lower tracing (May, 1955) abnormal $Q$ and $T$ waves have disappeared and the curve is within normal limits.

* Present address: Laikon Hospital, University of Athens, Greece. 
wave. After ligation of the artery the $\mathrm{R}-\mathrm{T}$ segment and $\mathrm{T}$ wave changes are reversible whereas the abnormal $\mathrm{Q}$ is permanent. In clinical cases, the cardiogram shows similar changes. After the acute phase of the infarction, the QRS-T complex gradually evolves to its permanent form; in many uncomplicated cases, the R-T segment and $\mathrm{T}$ wave become normal, the only residual abnormality being a deep $\mathrm{Q}$ or $\mathrm{QR}$ wave in leads where previously there was an $\mathrm{R}$ wave.

Experience has shown that while the abnormal Q wave is usually permanent (Wallace, 1934; Vander Veer and Edwards, 1939), rarely it may contract to normal dimensions, in which case the whole ventricular complex is normal. Little has been written on this point and few cases have been reported in which the cardiogram has returned to normal after the acute phase of a transmural infarction had subsided.

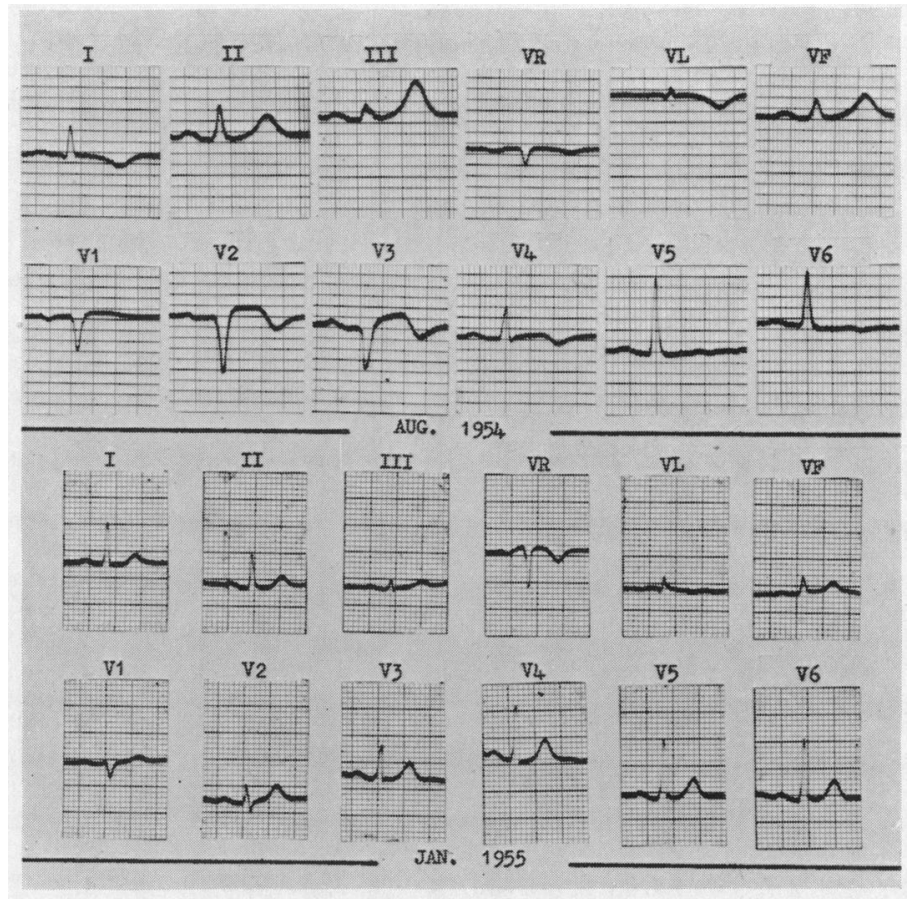

FIG. 2.-Signs of antero-septal infarction with ischæmic changes in the left chest leads were present in August, 1954. Five months later (January, 1955) the curve was normal.

Bohning and Katz (1938) followed up 200 cases of coronary occlusion over a period of three years and found only one in which the $\mathrm{Q}$ wave of a posterior infarction disappeared 18 months after the acute phase. Goldberger (1953) is of the opinion that an abnormal Q wave might occasionally diminish in size so that it could not be distinguished from a normal one and reports a case of posterior infarction in which it returned to normal dimensions in III and aVF after the acute episode, but emphasizes that it usually remains abnormal through life (Goldberger, 1955). Levine (1951) also believes that on rare occasions the abnormal Q wave disappears. On the other hand, Coelho et al. (1950) in a follow up of 320 cases of cardiac infarction do not mention even one instance in which the cardiogram returned to normal, no matter how the clinical condition had improved. The very few published instances of disappearance of abnormal $Q$ waves related to posterior infarctions; no example involving an anterior infarction has been reported so far.

In order to find out how often the QRS complex can revert to normal after healing of the necrosed area, we reviewed the cardiograms of 742 cases of cardiac infarction occurring between 1949-55 which were followed up in the Department of Cardiology of the Middlesex Hospital and 
discovered 14 cases $(1.9 \%)$ that seemed to have the necessary criteria. All were men between the ages of 49 and 66; six were above 55 and eight below, the average being 54 years. There was a short history of anginal pain before the infarction in all patients except two who had complained of pain for six months and two years respectively. The blood pressure in the majority was within normal limits. Nine patients had an antero-septal infarction, four had posterior infarction, and one had both. In one, the cardiographic signs of acute necrosis disappeared within a month. In all the others, reversion to normal took place between 2 and 18 months. Illustrative cases are shown in Fig. 1-4.

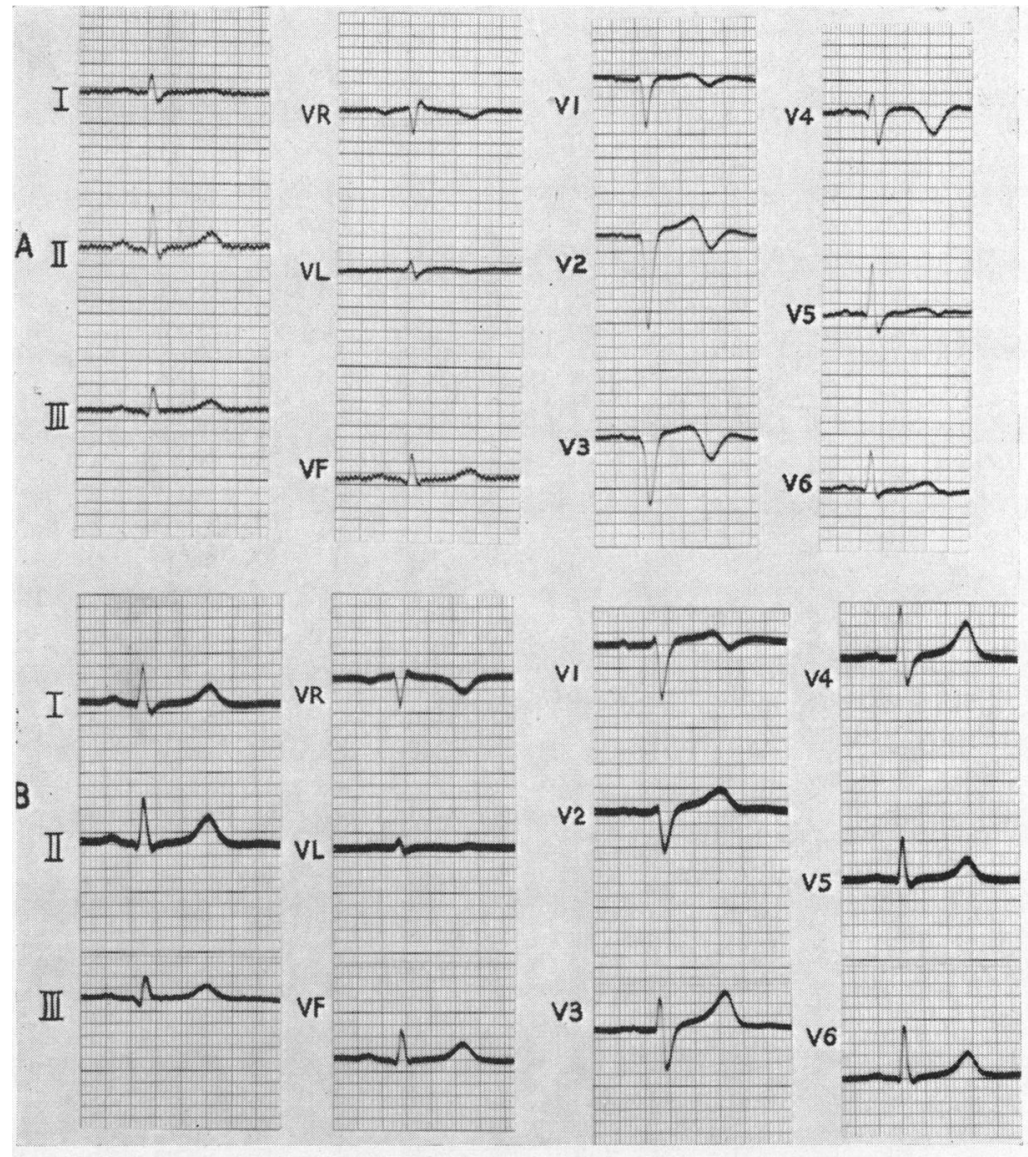

FIG. 3.-(A) Typical changes of antero-septal infarction are seen in leads V1-V4 in tracings taken in March and April, 1952. (B) In August, 1954, the cardiogram is normal.

When a pathological $\mathrm{Q}$ wave in III and aVF disappears or gives place to a small $\mathrm{q}$ of normal dimensions it is presumed that the scarred area has become too small to be detected by the limb leads in view of the wide angle subtended between these leads which must inevitably include a large portion of healthy myocardium. It is known that high posterior infarctions may not be detected by any of the conventional 12 leads. Shrinkage of the fibrous tissue in the posterior infarcts of the present series may have resulted in a higher localization than usual. Consequently a small $\mathrm{q}$ was recorded in leads III and aVF. But in two (Cases 6 and 8), the abnormal Q waves were replaced by small $r$ waves in the same leads (III and aVF) leaving no evidence of a previous posterior infarction. 
Holzmann (1952) points out that sometimes an abnormal Q wave disappears when a second infarction appears. In the case of Bohning and Katz (1938) the $Q$ of a posterior infarction was abolished when, after one and a half years, a new anterior infarction took place. In Cases 3 and 8 of our series the signs of an anterior infarction disappeared a few months after a fresh posterior infarction. Probably as the new infarction develops, a slight rotation of the heart masks the exact localization of the previously infarcted area. Levy and Hyman (1950) say the signs of a healed posterior infarction are occasionally difficult to differentiate from a normal tracing in a patient whose heart is in a horizontal, slightly clockwise-rotated position.

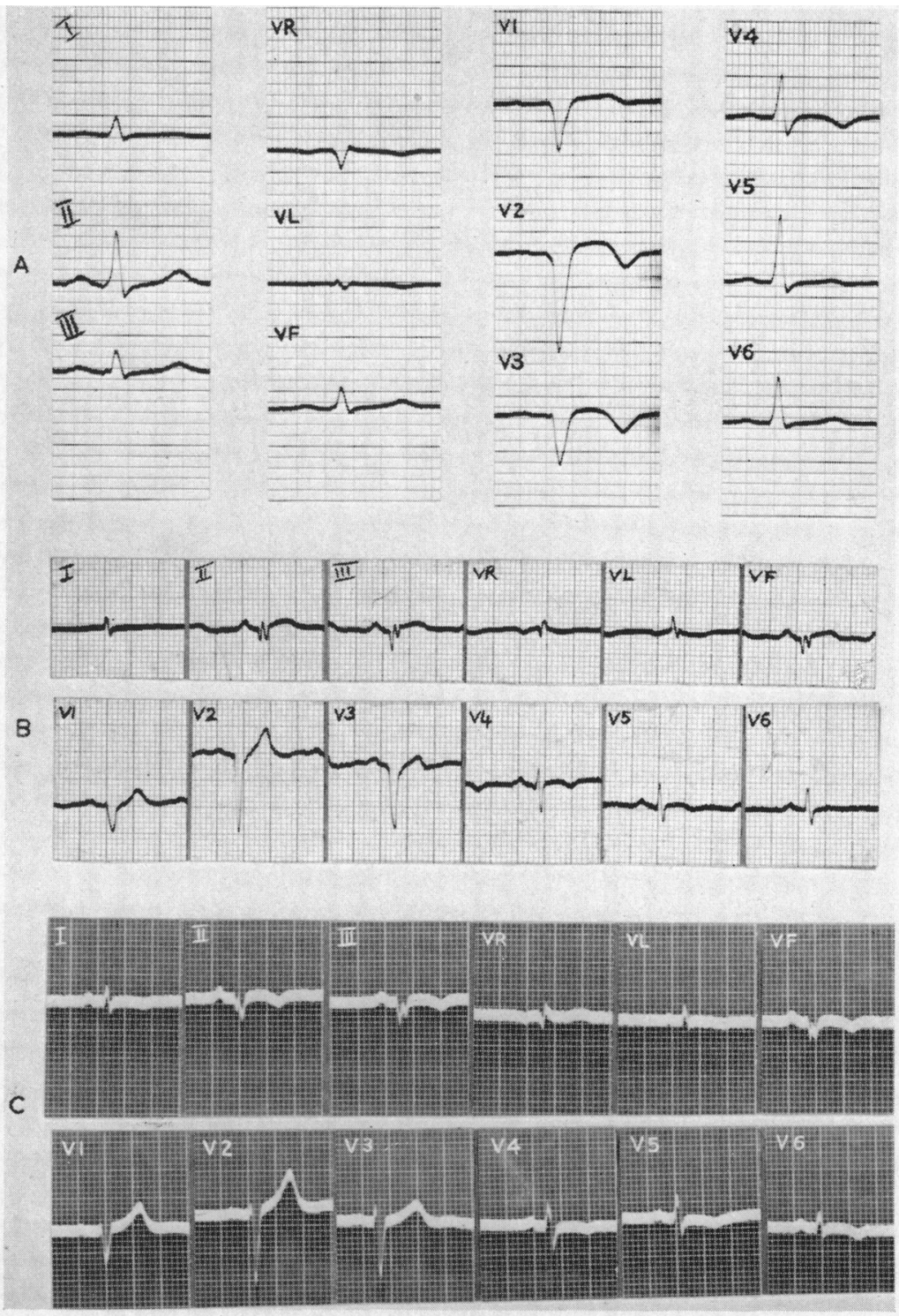

Frg. 4.-(A) Antero-septal infarction in January, 1953. (B) In February, 1955, posterior infarction has developed, with typical changes in leads II, III, and VF, the signs of antero-septal infarction persisting. (C) By March, 1955, the signs of antero-septal infarction have disappeared, but the posterolateral ischæmic changes still remain. 
But what has been said up to now concerns only posterior infarctions. The anterior and lateral walls of the heart are explored in more detail by the præcordial leads and it is hard to believe that a necrosed area here can disappear or that the abnormal $\mathrm{Q}$ waves in the chest leads can be replaced by $\mathrm{R}$ waves. Contratto et al. (1939) pointed out that the characteristic signs of infarction in the chest leads never disappeared in the cases they had followed. Vander Veer and Edwards (1939) agree that reversion to normal seldom if ever occurs.

In the case of antero-septal lesions, the explanation that has been given for the disappearance of the $\mathrm{Q}$ waves in posterior infarctions can also apply. Probably in these infarcts as well, the healed area has contracted in a part of the septum that is not detectable from the precordial leads and the myocardium reflected in V1-3 is activated in the normal manner. In recent septal infarctions the part of the septum responsible for the $\mathrm{r}$ waves ceases to contract and so a QS deflection appears in these leads. When healing is complete and the affected area has been so diminished in size that only the lower part of the septum is inert, excitation of the upper septum is virtually normal, with the result that $r$ waves reappear in the right chest leads.

Although similar pathological changes no doubt occur during healing of antero-lateral infarcts, leads V4-7 permit such detailed exploration of excitation in this area that the changes due to even small areas of necrosis are detectable. This probably explains the absence of any case in our series with antero-lateral infarction showing subsequent reversion to normality.

The reappearance of a normal cardiogram is no doubt related to the development of collateral circulation in the dead area. The failure of such a circulation to develop might explain why in other instances of antero-septal infarction there is no reversion to normal. In the present series the coronary arteries and their branches were presumably in a relatively healthy state before the occlusion and so the collateral circulation developed fully. Prinzmetal et al. (1948) have proved that even in the normal heart an abundant collateral circulation exists and a sudden obstruction of a major pathway permits the opening of preformed collateral channels. Possibly this collateral circulation can develop more readily in the antero-septal part of the myocardium, and this may explain some cases we have seen in which QS waves that were present in all chest leads from V1-V5 had become normal in V-V3 after some years although V4-5 still showed QS deflection. But not all the cases can develop the same degree of collateral circulation. Hecht (1949) showed two cases of infarction of the same branch of the left coronary artery demonstrated at autopsy: in one, a few years after the acute episode, the collateral circulation was almost perfect, whereas in the other it remained very poor. Does the collateral circulation develop to such an extent in these cases as to give a normal cardiogram? We have no necropsy confirmation.

\section{SUMMARY}

In a review of all cardiac infarctions seen in the Cardiac Department of the Middlesex Hospital between 1949-55 we have found 14 cases in which the abnormal ventricular complex of the electrocardiogram returned to normal. The infarction had been antero-septal in nine, posterior in four, and both antero-septal and posterior in one. No case of antero-lateral infarction reverted to normal.

All occurred in men with a short history of anginal pain, with the exception of two, who had angina of effort for six months and two years respectively. The blood pressure in most instances was within normal limits. The progress, in all the cases, after the infarction was satisfactory. The explanation is discussed and reasons are given for thinking that the return of the cardiogram to normal is due to the shrinkage of the healed area in a part of the myocardium not electrically detectable. A further factor may be the development of an efficient collateral circulation.

I am indebted to Dr. D. Evan Bedford for his encouragement in carrying out the present paper and to Dr. W. Somerville and Dr. E. M. M. Besterman for their criticism and advice. 


\section{REFERENCES}

Bohning, A., and Katz, L. N. (1938). Arch. intern. Med., 61, 241.

Coelho, E. Sousa, B.A., Bordalo, e sá, I. Barata, MI, and Maltez, J. (1950). Arch. bras. Cardiol., 3, 445.

Contratto, A. W., Robinson, R. W., and Levine, S. A. (1939). Arch. intern. Med., 63, 732.

Goldberger, E. (1953). Unipolar Lead Electrocardiography and Vectorcardiography. Kimpton, London. (1955). Heart Disease. Kimpton, London.

Hecht, H. H. (1949). Arch. intern. Med., 84, 711.

Holzmann, M. (1952). Clinical Electrocardiography. Staples Press, London.

Levine, S. A. (1951). Clinical Heart Disease. Saunders, London.

Levy, L., and Hyman, A. L. (1950). Amer. Heart J., 39, 243.

Prinzmetal, M., Bergman, H. C., Kruger, H. E., Schwartz, L. L., Simkin, B., and Sobin, S. S. (1948). Amer. Heart J., $35,689$.

Vander Veer, J. B., and Edwards, J. C. (1939). Amer. J. med. Sci., 197, 663.

Wallace, A. W. (1934). Amer. J. med. Sci., 188, 498. 